NASA Technical Memorandum 104390

AIAA-91-2325

\title{
Paramagnetic Propellant Orientation
}

R.C. Hendricks

Lewis Research Center

Cleveland, Ohio

Prepared for the

27th Joint Propulsion Conference

sponsored by the AIAA, SAE, and ASME

Sacramento, California, June 24-26, 1991

\section{N/SA}




\author{
R.C. Hendricks \\ National Aeronautics and Space Administration \\ Lewis Research Center \\ Cleveland, Ohio 44135
}

\section{Abstract}

Deep space or low earth orbital propellant tanks require a fluid orientation system prior to engine firing or transfer. Some propellants such as cryogenic hydrogen, oxygen, and ais are paramagnetic and respond to electromagnetic fields. Herein we describe a simple magnetic scheme for propellant orientation and provide a video tape presentation that demonstrates some effects of magnetic fields on liquids air and oxygen in a low gravity simulator using the Leidendfrost phenomenon. When these Leidenfrost drops intersect the field lines, their "flight" paths are altered, some directly into the poles, some to the edges, and others move out of the field.

Space storage of hydrocarbons for space manufacturing or other processes can be easily oriented using ferromagnetic colloids and some techniques described herein.

\section{Introduction}

In microgravity fields orientation of cryogens in storage tanks and settling prior to thruster firing is a major concern.

Currently surface tension driven devices such as fluid orientation screens and standpipes are used for propellant orientation. They rely entirely on surface tension which entails surface wetting or energies and thermal effects. The surface to volume ratio is critical to the orientation. Tank bumping, fountain effects from a jet driven fluid as well as supercritical pressure storage are some of several proposed schemes to solve the fluid orientation problem. ${ }^{1}$

Surface tension devices are simple but not always reliable as the attractive surfaces include the walls and standpipes that can be disabled. propellant bumping requires an impluse type disturbance, not good for transfer systems or stabilized platforms. Propellant fountains require active systems rather than passive with attendent reliability problems.

In this note we describe a simple reliable system using magnetic fields to orient some cryogens in space and provide a video tape presentation that demonstrates some effects of magnetic fields on liquids air and oxygen.

While it is unlikely that RP or its derivatives will be used for space missions due to its low Isp, there may be space manufacturing processes or space station requirements that need hydrocarbons. The hydrocarbons and cryogens have similar orientation problems. However hydrocarbon based fluids could be easily oriented using ferromagnetic colloids that do not interfere with the processes or environmental concerns. 2,3
Description of a Proposed Apparatus

The scheme proposed herein is a simple reliable system but is limited to paramagentic fluids, Fig. 1 .

So the first element of the system is a paramagentic cryogen such as liquids oxygen, air, and hydrogen.

The second element is the magentic field. Magnetic fields are created by either passive or active magnets. Passive are permanent types and active are electromagnetic. Some combinations with high temperature superconductors could be used with liquids oxygen and air while more conventional superconductors could be used with liquid hydrogen; superconducting fields have not been used herein.

The third element is a tailored grid or lattice structure fabricated to provide the desired magnetic field to attract and orient the paramagnetic propellant. For example the grid or lattice might be envisioned as a magnetic tree orientation system within a propellant tank. If the system has active magnets, then a control circuit is also required. While active controls can malfunction, they can be used to provide spacial and temporal gradients not possible with strictly passive systems.

While the system is simple to construct, the low gravity is not readily available and has not been done herein. However the first phase is to determine if fluids such as liquids oxygen and air that are proported to be paramagnetic can be attracted and controlled by magnetic fields using a low gravity simulation.

\section{Results}

Many substances exhibit diamagnetic or paramagnetic effects. Paramagnetic effects are associated with unpaired electrons which are attracted to a magnet; diamagnetic substances have paired electrons which are repelled by magnetic fields. Some magnetic properties of substances appear in Ref. 4.

To demonstrate that fluids air and oxygen are paramagnetic and could be controlled in microgravity, Leidenfrost droplets were used. The Leidenfrost droplet floats on a cushion of its own vapor which has a very small frictional resistance and as a result it is easily moved about the surface by small forces. When placed on a nearly flat surface the gravitational potential is uniform and the effects of other potentials are readily observed.

When determining the attraction or repulsion forces using the Leidenfrost simulator it is very important that the surface is not wetted. Because 
surface tension is low for cryogens, once the surface is quenched it wets very readily and the cryogen crawls all over the surface. Such behavior can be easily misconstrued as magnetic attraction.

In the video presentation Leidenfrost drops of enriched liquid air (LAIR) with an oxygen content of approximately 25-percent are attracted to a very small simple magnet typically used for holding notes on metallic substrates, Fig. 2 .

In Fig. 3, liquid oxygen is being confined between the pole pieces of a small horseshoe magnet (a simple demonstration appears on the video tape). The fluid that is in Leidenfrost film boiling on the surface of the magnet poles is not easily dislodged. However if the pole gap is sufficiently large, implying low field strength, liquid oxygen confinement is limited to the immediate pole neighborhood. Some very clear photographs of liquid oxygen held by magnetic fields are given by Profs. Zumdall and wilson.

To further eliminate materials, surface conditions, and surface temperature causing the attractive effects, one liquid air Leidenfrost drop experiment was repeated using a nonmagnetic material (stainless steel). There was no attraction to the nonmagnetic material.

If the field-fluid provides a gradient, then the fluid can be pumped. A simple demonstration of paramagnetic pumping appears on the video tape. While there are some surface tension effects they are readily overcome by the strength of the magnetic field. The field piece was dipped into the LOX on one end with the other side in the vapor. Lox filled the gap in between Liquid oxygen filled the gap and was pulled upward.

A paramagnetic fluid confined between the poles of a magnet and evaporating into a condensable environment provides some interesting physics and residual footprints. Liquid air was placed on a glass substrate and entrapped between the pole pieces of a horseshoe magnet.

In one case, the Leidenfrost drops go into their characteristic vibrational modes that are dependent of droplet volume, shape, residual vibrations, surface characteristics and temperature. Even though the field is present, the thermal perturbations are stronger and engender and drive the oscillations.

In another case, one can review the evaporation history of the confined paramagnetic fluid by studying residual "rings." The residue that circulates within the droplets and deposits on the surface are ices, carbon-dioxide and water from the air environment. With proper backlight the glass and you can see the characteristic rings associated with the droplet evaporation; and at the pole pieces more concentrated residuals look like little eyelets, Fig. 4. The halo or elliptical pattern around the outside perimeter is from the water vapor condensed from the atmosphere by the cold vapor flowing from beneath the evaporating droplet.

\section{Video Presentation}

The accompanying video tape is entitled A Demonstration of Paramagnetic LAIR and LOX Orien- tation by Electromagnetic and Magnetic Field Effects.

A transcript of the tape audio is given in the appendix. The tape covers simple attraction of liquids air and oxygen, shows that liquid nitrogen is not paramagentic (as it is diamagnetic), demonstrates a simple electromagnet, illustrates some residual patterns from fluid evaporation from a glass substrate, the attraction of paramagnetic Leidenfrost drops streaking across a lucite plate in a magnetic field, and provides a section on applications that are conceptual and require further development.

The preliminary video tape, representing a composite of several video tapes, was completed and presented to management in May 1989 and has engendered recent interests in paramagnetic flowmetering and propellant orientation. 6,7 And I must thank Robert Zubrin for calling my attention to a paper on interface deformation of liquid oxygen in high magnetic fields.

\section{Conclusions}

Paramagnetic cryogens liquid air and oxygen are attracted to and confined within the poles of a magnet.

Active or passive magnetic fields imposed on paramagnetic fluids could be used to provide a deep space or low earth orbital propellant tank fluid orientation system.

Field-fluid gradients could be used to pump paramagentic cryogens.

"Ring" residuals from evaporated Leidenfrost paramagentic fluids provides a visual image of the confinement history through concentration of deposits.

Under space conditions ferromagnetic colloids might be used to orient hydrocarbons.

\section{Appendix}

A Transcript of the Audio for the Video/Audio Presentation Entitled

Electromagnetic and Magnetic Field Effects A Demonstration of Paramagnetic LAIR and LOX Orientation by

Electromagnetic and Magnetic Field Effects

R.W. Hendricks

Wilson Magnet School Rochester, New York

R.C. Hendricks

NASA Lewis Research Center Cleveland, OH 44135

Simple Magnetic Attraction of Enriched LAIR in Film Boiling

Many substances exhibit diamagnetic or paramagnetic effects. Paramagnetic effects are associated with unpaired electrons which are attracted to a magnet. With diamagnetic effects the electrons are paired and are repelled by magnetic fields. 
Here we have a Leidenfrost drop, in this case enriched liquid air (LAIR) in which the oxygen content of this droplet is approximately 25-percent. The droplet is attracted to a very small, simple magnet (for instance the kind used to hold paper onto a refrigerator door). The Leidenfrost droplet floats on a cushion of its own vapor which has a very small frictional resistance and as a result it is easily moved about the surface by small forces. You can draw or drop the magnet through it, in this particular case, without wetting the surface of the magnet. When determining the attraction or repulsion forces it is very important that the surface is not wetted because surface tension is low for cryogens like LAIR and once the surface is quenched it wets very easily and the cryogen crawls all over the surface. Such behavior can be easily misinterpreted as magnetic attraction.

\section{No Attraction to NonMagnetic Materials}

In the case of nonmagnetic materials, the paramagnetic fluid is not attracted to the surface at 211 unless it is wetted. In this case a nonmagnetic material, stainless steel, can be drawn through the paramagnetic fluid without any attraction whatsoever.

Here the small magnet is brought back into the configuration, and fluid orientation is usually attained by the magnet holding the droplet in place wherever you want. You can even see some of the paramagentic fluid being lifted off the surface by the magnet.

The residue seen within the droplets are ices, carbon-dioxide, and water from the air environment. Water vapor is very prominent here with a little droplet or ice ball that's floating around in a circle. Since ice segregates charge and holds charge in a field, the attractive effect could be due to a dipole such as is the case for a colloid in ferromagnetic materials. But in this particular case the ice ball rotates freely within the droplet as the droplet in turn is being attracted by the magnet. The droplet surface is being heated from below by the metallic interface as well as from above by the environment. The cold vapors from the droplet condense out some of the water vapor in the air but also gather water vapor from the interface as the droplet travels across the metallic surface. It is interesting to note that incipient oxygen condensation can occur. If the droplet were liquid nitrogen then some oxygen condensation would occur and must be ruled out during magnetic studies of liquid nitrogen.

\section{Electromagnetics (Cryogenic) A.C. Power}

Here we have an electromagnet. Electromagnetic field effects, in this case from alternating current, hold the droplet in place very nicely. The Leidenfrost drop tends to vibrate. This is characteristic of a Leidenfrost drop. There are several modes of vibration depending on the volume, heating, surface tension, void, and things like that. But here note that the electromagnetic field does not inhibit the vibration but simply tends to orient the fluid under the tip of the cryomagnet. In this case the magnet was dunked in liquid nitrogen prior to holding it over the drop. Precooling gives it increased field strength but you have to be very careful because the drop can wet the surface and once it wets, the liquid layer will crawl all over the magnet surface, resulting in the kind of misleading attraction cited earlier.

\section{Electromagnets (heated coils) Alternating Current}

Here the magnet has been heated and as you will see later on in this sequence the tip of the magnet will eventually get above the freezing point of water and the moisture that has been collected at the tip will disappear. And again I have to caution that once the fluid wets the surface the surface properties of this experiment are such that the droplet will cool it very rapidly in nucleate boiling where heat transfer rates are high rather than remaining in film boiling where the heat transfer rates are very low.

\section{Horseshoe Magnet}

This horseshoe magnet orients the fluid very nicely. Only a small amount of fluid is used here as it takes quite a while for it to vaporize by film boiling from a glass substrate. The droplets are held near the ends of the pole pieces and of course they go into their characteristic vibrational modes. The residue that forms here will of course be the ices from water vapor and carbon dioxide.

Once the trapped fluid has evaporated, the residuals leave patterns. The picture shown here illustrates a very beautiful pattern. once you properly backlight the glass and you can see the characteristic rings associated with the droplet evaporation, the pole pieces that look like little eyelets here. The halo or elliptical pattern around the outside perimeter is from the water vapor condensed from the atmosphere by the evaporating droplet.

Dr. Jerry Brown measured the field strength as follows: Max field $3.9 \mathrm{k}$ gaus toward inside pole center; $3.6 \mathrm{k}$ gaus at the pole and $2.8 \mathrm{k}$ gaus in the center.

\section{Capturing and Confinging LOX}

Here we have Lox itself. Liquid oxygen is being held between the pole pieces of a small magnet. The magnet surface again is quite warm. In other words, it's a little cooler than room temperature, but you can see the liquid oxygen being held between the poles pieces of the magnet; the excess, of course, runs off. But Lox, or liquid oxygen, is film-boiling from the faces of the magnet as it is being held by the magnetic field. If the pole gap is sufficiently large, of course, you can not lift the liquid oxygen, but here it is easily pulled off the surface. The gap shown here is completely filled with liquid oxygen and again the excess runs off. Clearly here it is held between the pole pieces of the magnet. Again, it is important that you do not quench or bring the surface below the Leidenfrost temperature because the cryogens will wet the surface and crawl all over it rapidly and you cannot control it as easily as you did before. It also can be misleading and be interpreted as magnetic attraction when it is simply a surface tension phenomena. The reason you can hold it there is that the liquid oxygen or Lox is in a state of film boiling and easily controlled.

Here is a little different view of what's going on, while the fluid is being pumped. It is 
being pulled a little by surface tension and at the same time by the strength of the magnetic field. The field piece is dipped into the Lox on one end and in the vapor on the other end. LOX fills the gap in between and pulls itself into position as shown particularly well here. Lox fills the bottom of the magnet gap and climbs up through the gap; again the pull is a combination of surface tension and magnetic field but principally its the magnetic field. As you can see, small forces like these magnetic forces will orient the fluids.

\section{Attracting LOX in a Saturated Swab}

In this particular case, if you take a cotton swab or q-tip and you dunk it into the cryogen, which is a standard procedure in dermatological applications, the swab will be attracted to the magnet until, of course, the LOX vaporizes out of the swab and it drops from the magnet to the ground or surface.

\section{Q.E.D}

These experiments were set up to demonstrate that paramagnetic fluids such as LAIR (liquid air) and LOX (1iquid oxygen) are attracted to the poles of a magnet.

\section{Applications}

As with any new phenomenon, or one which you think is new, you begin to consider ways to apply it. You think of schemes and experiments that you would like to try such as trying to figure out the magnetic susceptibility of a paramagnetic fluid (PMF) by suspending it between the poles of a magnet.

In this particular case, you might want to actually take a drop and suspend it between the poles of an ordinary magnet. Here PMF is the paramagentic fluid and $B$ is the control field on the magnet itself which is necessary to fine tune the suspension field between the magnet and the PMF droplet which provides the suspension.

You might want to suspend the PMF between the poles of a cryogenic magnet, but as you recall from our previous discussion, when the PMF and the poles of the magnet are roughly the same temperature, the PMF wets the surface and tends to crawl all over it (ideal for space fluid orientation discussed later). This will make a difference in your suspension system. So you might want to inject a buffer gas such as helium between the poles of the magnet.

You might want to extend this concept to the case where you have a fluid sump or a standpipe. The magnets may be high temperature superconducting magnets or in the case of hydrogen, you might want to use conventional magnetic materials and standard superconductors with higher fields, or you may just want to use the conventional permanent magnets and judiciously distribute them in some network about the standpipe or sump. The sump would be the area for collecting the fluid for the pump in a low gravity environment, for instance.

These fields can also be used to prevent stratification of fluid mixtures containing paramagnetic components or conversely can be used to provide stratification or separation of paramagnetic mixtures.

You might even want to extend this further to the case in which you would actually confine the PMF in the entire region instead of just in a standpipe. We think of a magnetic thermos bottle simply having a gas layer or buffer layer on the periphery near the walls of the tank and the paramagnetic fluid within the envelope. This is just like film boiling or the Leidenfrost phenomena, and characterizes high heat flux hydrogen heat transfer, that is the hydrogen gas is next to the heated surface with the liquid in the core. The thermos bottle concept would be interesting as it would cut down the heat leaks to your PMF, or fluid, confined in this region. Again, you might want to use high temperature superconducting magnets or you might want to use conventional superconductors at lower temperatures, or conventional magnets on some type of frame or array.

Actually, there are many simulations that could be done in the one-gravity environment because Leidenfrost droplets scoot around on a cushion of their own vapor and as such are affected by fairly small forces. The field strengths here are such that they are small forces. The magnetic field is related to the electric field through the velocity of light and that illustrates the low strength of the magnetic field. The Leidenfrost drop that you have here might be in a field that is contoured both in time and space. Given a timevarying field, you can contour the strength of the field to give your field a space variation; so you would have the possibility of studying PMF fluids in both space and time in this type of flat plate simulator.

Studies in chaos are another aspect of this phenomena. If a drop is approaching a magnet, as in the upper part of the picture of the video picture, the droplet can either go to the lower part of the picture or to the upper part of the picture, depending on the initial conditions in which the drop finds itself in this magnetic field. If there are small perturbations in the droplet, it might go to the lower part of the picture or the upper. Suppose the perturbation is such that it goes to the upper part of the picture. Similarly to the one-g simulator we discussed before, you can enable the droplet to enter an array of magnets or a contoured field which is defined in space. You can also vary it in time by cycling the magnets on and off or perturbing them so the field strength changes with various time intervals. With droplets approaching with different perturbations, you can then study the effects of the drop as it goes through this magnetic field. The perturbations and drops could be random or ordered. This configuration provides a good simulation of some of the chaotic states currently under study.

Parahydrogen if also paramagentic and as such the above fipdings would also apply to parahydrogen. 20

\section{Systems}

If you look at these phenomena from a bigger point of view perhaps you might see things like magnetic confinement or fluid stability of a PMF as studied by many people for the fusion type 
processes. The problem of magnetic confinement is difficult, and the standard fusion process to which I refer does not necessarily preclude the cold fusion process. Here we offer a reasonable simulation of the confinement problem and $a$ way to study it under fairly well-defined conditions.

Taking this idea a little further, let the PMF be encapsulated or simply suspended in the field of a baseball magnet and surrounded by a region of fluid hydrogen. The PMF could be a slush or type of pellet, for instance, to provide the fuel/ oxidizer combination. In a controlled reaction the energy release would be slow and regular. If you had a rapid release and if the PMF is LOX, and LOX and parahydrogen like each other a lot, you would have rapid release of energy.

Another system that could be studied is one that contains a reentry type body. In this system you have a flow envelope of ions which are fairly long lived and affected by a magnetic field. A flow of ions is a current and if you do have a magnetic field you can envelope the body with this magnetic sheath. You can cool the magnets, for instance, by force convection cooling and you could use standard or high temperature superconducting magnets in most instances. In another way you can bury the coils of the magnet in your propellant tank, which acts as the heat sink, and which could also act to hold the coolant to the coils through their paramagnetic effects.

In some cases an effective seal or switch can be implemented. For instance in a rotating system magnetic fields can hold a paramagnetic fluid in place in the disc cavity at low rotational speeds but as the speed increases, the fluid is centrifuged of the disc into a outer passage forming a seal. Since the shroud materials are nonmagnetic the fluid would be returned to the magnet on the disc at low rpm. This is also the substance of a switch as in a fluid amplifier whereby the fluid path is switched by activating a magnetic field. Measuring instruments could be fabricated using this same effect.

Other things come to mind such as the study of the relation between the magnetic field and electromagnetic waves. This is the way I feel the field is formed. The other things you may be interested in might be the mobility of the oxygen ions in the higher temperature superconductors and, of course, pumping. PMF and other types of magnetic fluids can be pumped.

These are concepts and few experiments have been done so a lot more work remains to be done.

Roxy Wilson has a very nice experiment with LOX and Steve Zumdall has some nice pictures at the University of Illinois.

\section{References}

1. Aydelott, J.C. and Devol, W., eds., Cryogenic Fluid Management Technology Workshop. Vol. 1Presentation Material and Discussion, NASA CP-10001; Vol. 2 Roundtable Discussion of Technology Requirements, NASA CP-10009, 1988.

2. Papel1, S.S. and Faber, O.C., Jr., "On the Influence of Nonuniform Magnetic Fields on Ferromagnetic Colloidal Sols," NASA TN-D-4676, 1968 .

3. Second International Conference on Magnetic Fluids. IEEE Transactions on Magnetics, Vol. MAG-16, No. 2, Mar. 1980.

4. Bioudreaux, E.A. and Mulay, L.N., Theory and Applications of Molecular Paramagnetism, WileyInterscience Pub., New York, 1976.

5. Wilson, Roxy and Zumdall, S., University of Illinois, private communication, 1989.

6. Millis, M.G., Bewley, D., and Hendricks, R.C., "Paramagnetic Flow Meter for Cryogenic Propellants, Propellant settling and Alternate Flowmeter Research," Presentation of space Propulsion Technology Division, NASA Headquarters, Feb. 20, 1991.

7. Zubrin, R., Martin-Marietta Corp., Denver Colorado, private communication, Mar. 1991.

8. Takeda, M. and Nishigaki, K., "Shape deformation of the gas-liquid interface of liquid oxygen in high-magnetic fields," Physical Review A, Vol. 43, No. 4, Feb. 15, 1991, pp. 2081-2083. 
Propellant tanks in Zero-G

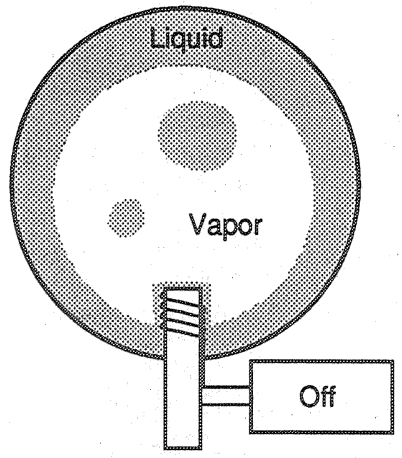

When solenoid is off, vapor can exist at pipe

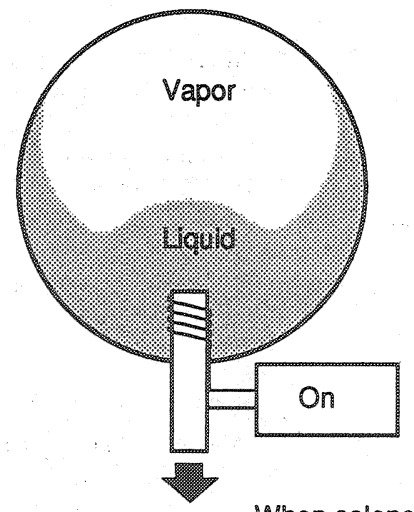

When solenoid is on, liquid is pulled into pipe

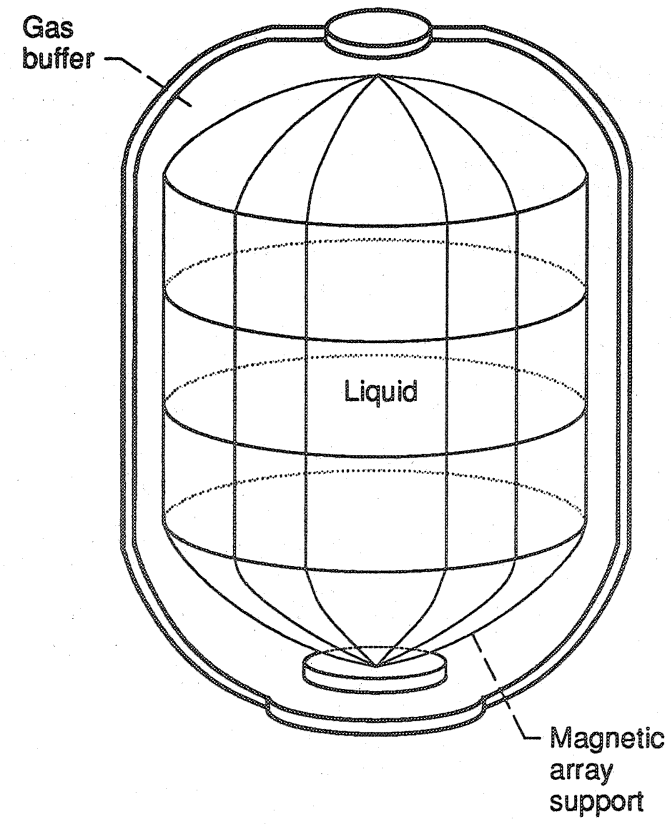

Figure 1.-Paramagnetic fluid orientation schemes for microgravity environments.

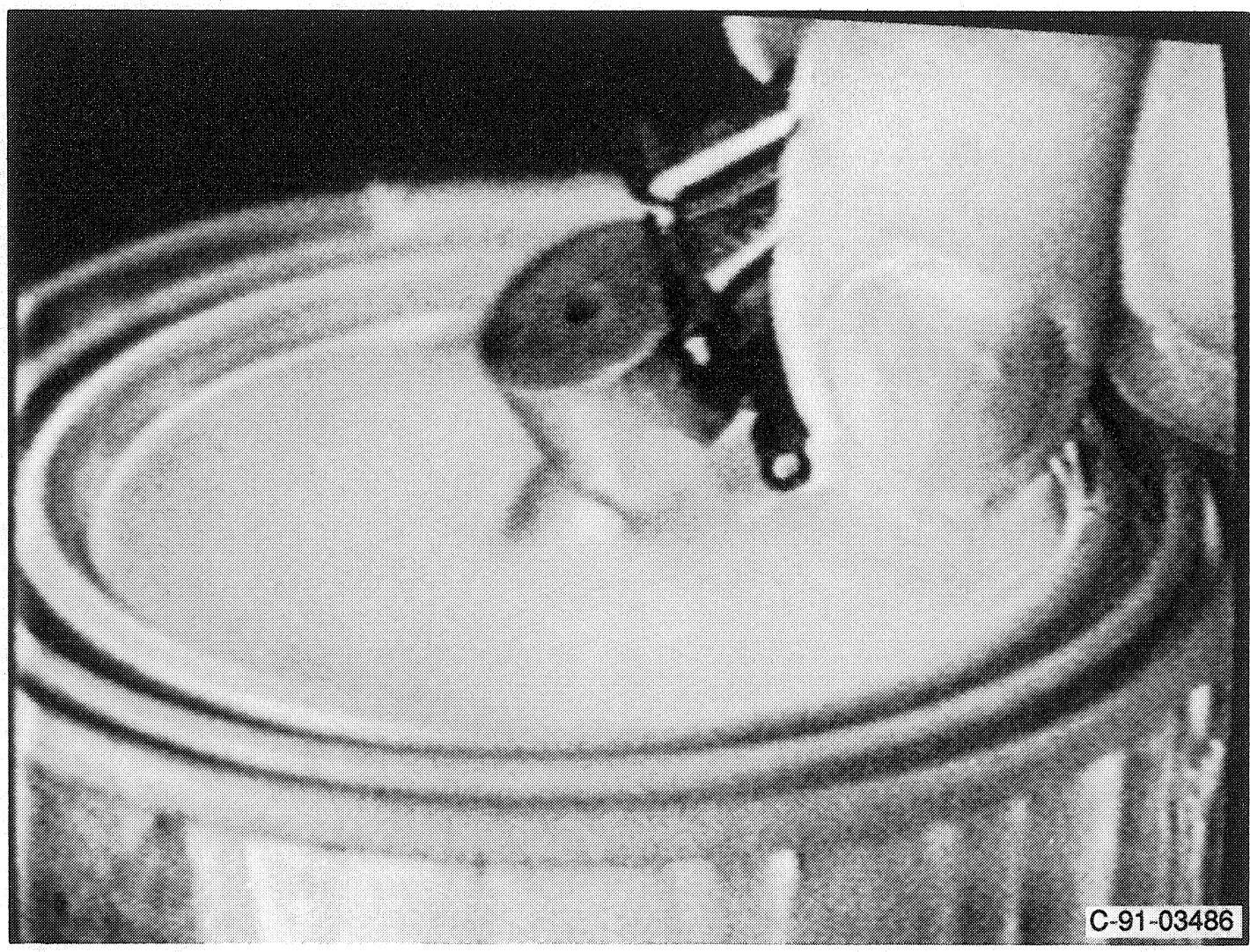

Figure 2.-Liquid air attracted to a simple magnet. 


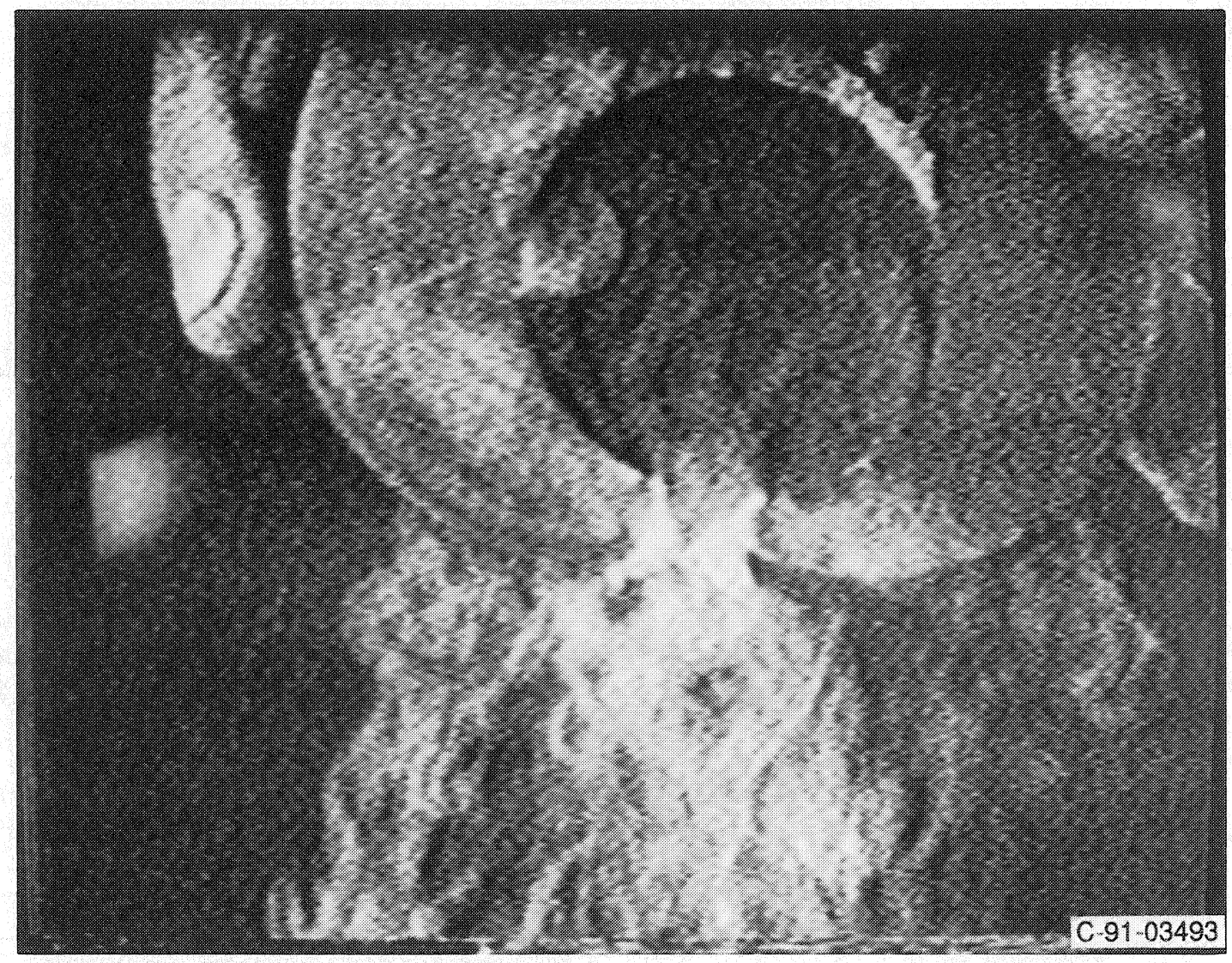

Figure 3.-Liquid oxygen held between the poles of a horseshoe magnet.

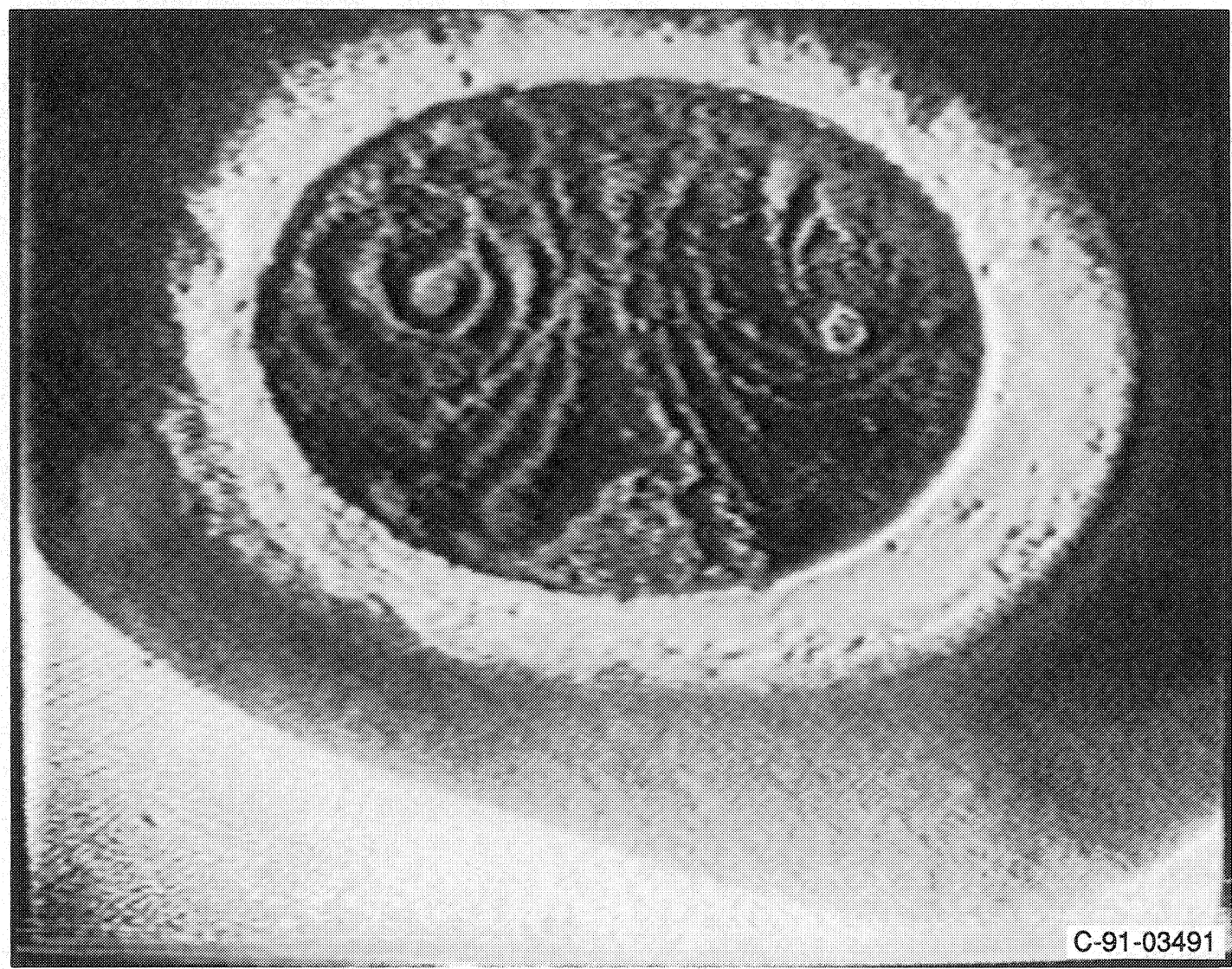

Figure 4.-A residual pattern formed by the evaporation of liquid air confined by a magnet. 


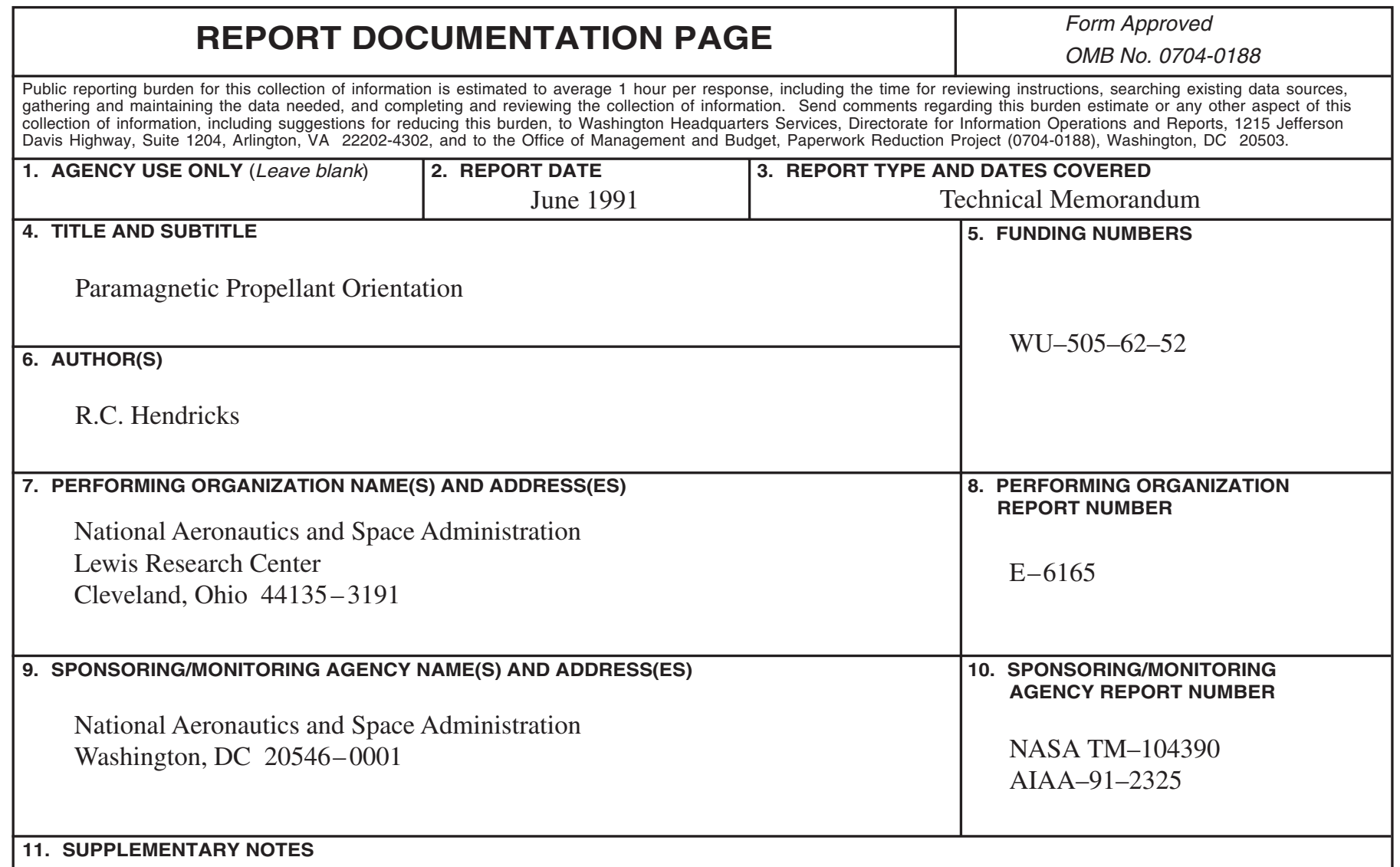

Prepared for the 27th Joint Propulsion Conference cosponsored by the AIAA, SAE, and ASME, Sacramento, California, June 24-26, 1991. Responsible person, R.C. Hendricks, 216-433-5912.

\begin{tabular}{|l|l|}
\hline 12a. DISTRIBUTION/AVAILABILITY STATEMENT & 12b. DISTRIBUTION CODE
\end{tabular}

Unclassified - Unlimited

Subject Category: 34

Available electronically at http://gltrs.grc.nasa.gov

This publication is available from the NASA Center for AeroSpace Information, 301-621-0390.

\section{ABSTRACT (Maximum 200 words)}

Deep space or low earth orbital propellant tanks require a fluid orientation system prior to engine firing or transfer. Some propellants such as cryogenic hydrogen, oxygen, and air are paramagnetic and respond to electromagnetic fields. Herein we describe a simple magnetic scheme for propellant orientation and provide a video tape presentation that demonstrates some effects of magnetic fields on liquids air and oxygen in a low gravity simulator using the Leidenfrost phenomenon. When these Leidenfrost drops intersect the field lines, their "flight" paths are altered, some directly into the poles, some to the edges, and others move out of the field.

\begin{tabular}{|c|c|c|}
\hline \multicolumn{3}{|l|}{ 14. SUBJECT TERMS } \\
\hline $\begin{array}{l}\text { 17. SECURITY CLASSIFICATION } \\
\text { OF REPORT } \\
\text { Unclassified }\end{array}$ & $\begin{array}{l}\text { 18. SECURITY CLASSIFICATION } \\
\text { OF THIS PAGE } \\
\text { Unclassified }\end{array}$ & $\begin{array}{l}\text { 19. SECURITY CLASSIFICATION } \\
\text { OF ABSTRACT } \\
\text { Unclassified }\end{array}$ \\
\hline
\end{tabular}

